\title{
Soil-Pile Dynamic Interaction in the Viscous Damping Layered Soils
}

\author{
Yinhui Wang ${ }^{1}$, Kuihua Wang ${ }^{2}$, Zhixiang Zha ${ }^{1, *}$ and Renbo Que ${ }^{2}$ \\ ${ }^{I}$ Department of Civil Engineering and Architecture, Ningbo Institute of Technology, Zhejiang University, Ningbo, \\ 315100, China \\ ${ }^{2}$ MOE Key Laboratory of Soft Soils and Geoenvironmental Engineering, Zhejiang University, Hangzhou, Zhejiang, \\ 310058, China
}

\begin{abstract}
Modeling surrounding soil as a three-dimensional axisymmetric continuum and considering its wave effect, soil-pile dynamic longitudinal interaction in viscous layered soils is studied. The pile is assumed to be vertical, elastic and of uniform section, and the soil is layered and visco-elastic. Longitudinal vibration of pile in viscous damping layered soils undergoing arbitrary load is theoretically investigated. By taking the Laplace transform, the question can be solved in frequency domain. Utilizing two potentials combined with impedance transfer functions, analytical solutions for both the impedance function and mobility at the pile head in frequency domain are yielded. With the convolution theorem and inverse Fourier transform, a semi-analytical solution of velocity response in time-domain undergoing a half-cycle sine pulse force is derived. Based on the solutions proposed herein, the effects of variety of soil modulus on mobility curves and reflection wave curves are emphatically discussed. The results shows that there is a smaller peak between every two adjacent larger peaks on the mobility curve in layered soil, and larger peak cycle reflects the location where the modulus of the soil varies abruptly. The conclusions can provide theoretical guidance for non-destruction test of piles.
\end{abstract}

Keywords: Soil-pile dynamic interaction, Layered soils, Viscous damping, Admittance curves, Mobility curves, Reflection wave curves.

\section{INTRODUCTION}

Dynamic non-destruction test method based on the pile vibration theory is widely used to identify pile integrity in civil engineering. Many soil-pile dynamic interaction models have been developed to simulate the behavior of longitudinal vibration of pile. From the view point of different model of the surrounding soil, they can be put into two categories, that is, Winkler model [1-7] and continuum model [8-13]. In the former model, soil is modeled by the distributed Voigt body. However, the value of parameters in Winkler model, that is, stiffness of spring and damping of dashpot, can't correlate well with the usual soil testing result. What's more, it is difficult to consider the wave effect of the surrounding soil. The first kind of continuum model [8-11] is Plain-Strain model with assumption that soil consists of independent infinitesimally thin layers extending to infinity horizontally. In Plain-Strain model, it is assumed that the gradient of strain and stress in the vertical direction is zero and the waves propagate only horizontally. Such assumption does not match well with reality. The second kind of continuum model $[12,13]$ takes the gradient of stress of the surrounding soil in vertical direction into account and hence can consider the wave effect of the surrounding soil. Nevertheless, two import factors, say, the radial displacement and the

*Address correspondence to this author at the Department of Civil Engineering and Architecture, Ningbo Institute of Technology, Zhejiang University, Ningbo, 315100, China; Tel: +86-574-88229069;

Fax: +86-574-88229587; E-mail: zhazx71@126.com axisymmetric wave effect of the surrounding soil, are all neglected in above models. Furthermore, the foundation soil is layered and this layered nature has a significant impact on the dynamic response of the pile and hence should be considered.

Based on above review, the purpose of this paper is to derive an analytical solution for longitudinal vibration of pile subjected to harmonic longitudinal excitation in layered soils by taking the axisymmetric wave effect of the surrounding soil into account. Utilizing the solution derived herein, the effects of various soil parameters on the longitudinal vibration of pile are discussed.

\section{FORMULARION}

\subsection{Pile-Soil System Model}

The problem studied herein is the longitudinal vibration of pile embedded in a layered soil with viscous damping. The geometric model is shown in Fig. (1). The soil-pile system is discretized into a total of $n$ layers numbered by $1,2, \ldots, n$ from the pile toe to pile top. The properties of pile and soil layer are assumed to be homogeneous within each layer respectively, but may vary from layer to layer. In the $k$ th soil-pile layer $(1 \leq k \leq n)$, the mass density, modulus of compression, thickness, poisson's ratio, longitudinal wave velocity, transversal wave velocity of the soil are denoted by $\rho_{s k}, E_{s k}, h_{k}, v_{k}, V L_{k}$ and $V S_{k}$, respectively. The Lame constants are $\lambda_{k}$ and $\mu_{k}$ and the corresponding viscosity 


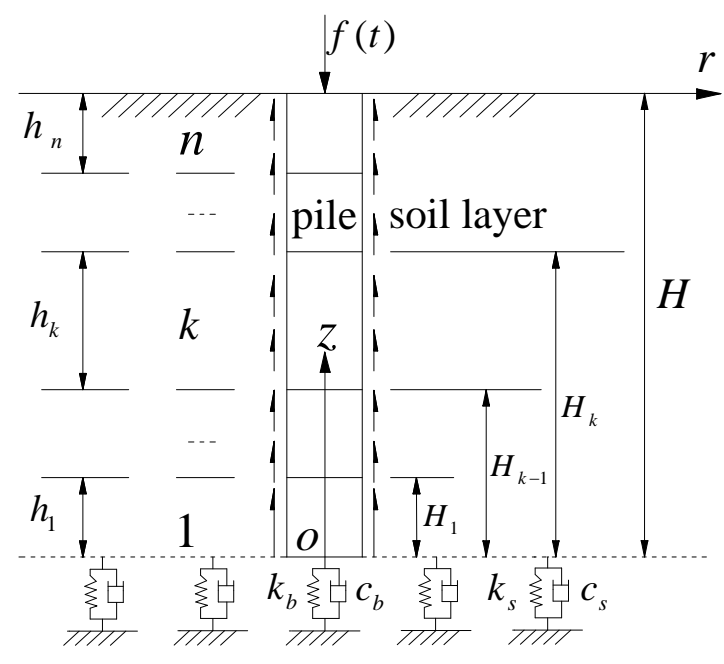

Fig. (1). Schematic illustration of pile-soil system.

coefficients are $\lambda_{k}^{\prime}$ and $\mu_{k}^{\prime}$, respectively. Here $E_{s k}=2\left(1+v_{k}\right) \mu_{k}$ and $\lambda_{k}^{\prime}=\frac{2 v_{k}^{\prime}}{1-2 v_{k}^{\prime}} \mu_{k}^{\prime}$, where $v_{k}^{\prime}$ is the transverse ratio of the viscous deformation rate. The length, mass density, elastic modulus, longitudinal wave velocity, cross sectional area and sectional radius of the $k$ th pile layer are expressed as $H, \rho_{p}, E_{p}, V P, S$ and $r_{0}$, respectively. $f(t)$ is an arbitrary vertical exciting force acting on the pile top and $R_{k}(z, t)$ is a friction force acting on the surface of the pile along per unit length in the $k$ th layer. The support of soil at the toe of pile is described as a single Voigt body which consists of a linear spring and a dashpot connected in parallel. The elastic modulus of the linear spring and the damping coefficient of the dashpot are denoted by $k_{b}$ and $c_{b}$, respectively. The supports of surrounding soil at the level of pile toe are described as distributed Voigt bodies in radial direction. The elastic modulus of the linear spring and the damping coefficient of the dashpot are expressed as $k_{s}$ and $c_{s}$, respectively.

\subsection{Assumptions}

The soil-pile system model is developed on the basis of the following assumptions:

(1) The surrounding soil layers with linear viscoelastic damping are assumed to be isotropic and homogeneous within each layer. The soil is infinite in the radial direction with free boundary condition at the surface of the soil. The soil radial displacement at the interface of the pile shaft is regarded to be small and thus can be neglected.

(2) The excitation is harmonic. The soil-pile system is subjected to small deformations and strains during the vibration. Pile and soil contact perfectly and therefore both force equilibrium and displacement continuity are satisfied at the interface of soil-pile, soil-soil and pile-pile.
(3) The pile is elastic and vertical with uniform circular cross section.

(4) The initial displacement and velocity in the soil and pile are zero.

\subsection{Dynamic Equation of Soil}

The geometric model of soil-pile in $k$ th soil layer is shown in Fig. (2). The support of the surrounding soil at the top and bottom are described as Voigt bodies distributed in radial direction. The elastic modulus of the linear spring and the damping coefficient of the dashpot are denoted by $k_{s s k}$, $c_{s s k}$ and $k_{s x k}, \quad c_{s x k}$, respectively. The vibration is axisymmetric. Let $u_{r k}(r, z, t), u_{z k}(r, z, t)$ to be the radial and vertical displacement, respectively and then dynamic equation of soil can be written as follows:

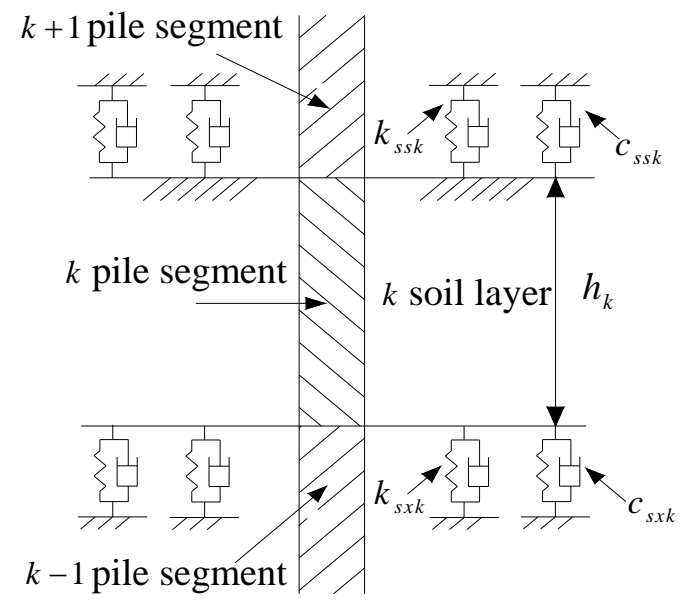

Fig. (2). Schematic illustration for k soil layer.

Radial direction:

$G_{1 k}\left(\nabla^{2}+\frac{1}{r^{2}}\right) u_{r k}+2 G_{2 k} \frac{\partial}{\partial z} \omega_{\theta k}=\rho_{s k} \frac{\partial^{2} u_{r k}}{\partial t^{2}}$

Vertical direction:

$G_{1 k} \nabla^{2} u_{z k}-2 G_{2 k}\left(\frac{\partial}{\partial r}+\frac{1}{r}\right) \omega_{\theta k}=\rho_{s k} \frac{\partial^{2} u_{z k}}{\partial t^{2}}$

where $G_{1 k}=\left(\lambda_{k}+2 \mu_{k}\right)+\left(\lambda_{\kappa}^{\prime}+2 \mu_{\kappa}^{\prime}\right) \frac{\partial}{\partial t}$,

$G_{2 k}=\left(\lambda_{k}+\mu_{k}\right)+\left(\lambda_{k}^{\prime}+\mu_{k}^{\prime}\right) \frac{\partial}{\partial t} \quad, \nabla^{2}=\frac{\partial^{2}}{\partial r^{2}}+\frac{1}{r} \frac{\partial}{\partial r}+\frac{\partial^{2}}{\partial z^{2}}$ and

$\omega_{\theta k}=\frac{1}{2}\left(\frac{\partial u_{z k}}{\partial r}-\frac{\partial u_{r k}}{\partial z}\right)$.

\subsection{Dynamic Equation of Pile}

Assuming the pile to be a one-dimensional continuum, its dynamic equation can be expressed as: 


$$
E_{p} S \frac{\partial^{2} w_{k}(z, t)}{\partial z^{2}}+R_{k}(z, t)=m \frac{\partial^{2} w_{k}(z, t)}{\partial t^{2}}
$$

where $m$ and $w_{k}(z, t)$ are mass of unit length and vertical displacement, respectively.

\subsection{Boundary Conditions and Initial Conditions}

For convenience, local coordinate system is adopted herein. This means that the coordinate is zero at the bottom and $h_{k}$ at the top in the $k$ th soil-pile layer.

(1) Boundary conditions of the soil layer:

(1) Displacements approach zero at an infinite radial distance:

$\left\{\begin{array}{l}\left.u_{r k}(r, z, t)\right|_{r \rightarrow \infty} \rightarrow 0 \\ \left.u_{z k}(r, z, t)\right|_{r \rightarrow \infty} \rightarrow 0\end{array}\right.$

(2) Radial displacement is zero at the interface of soil and pile:

$u_{r k}\left(r_{0}, z, t\right)=0$

(3) At the bottom of the $k$ th soil-pile layer, stress boundary condition is as follows:

$\frac{k_{s x k}}{E_{s k}} u_{z k}+\frac{c_{s x k}}{E_{s k}} \frac{\partial u_{z k}}{\partial t}-\left.\frac{\partial u_{z k}}{\partial z}\right|_{z=0}=0$

where $k_{s x 1}=k_{s}, c_{s x 1}=c_{s}$.

(4) At the top of the $k$ th soil-pile layer, stress boundary condition is as follows:

$\left\{\begin{array}{l}\frac{k_{s s k}}{E_{s k}} u_{z k}+\frac{c_{s s k}}{E_{s k}} \frac{\partial u_{z k}}{\partial t}+\left.\frac{\partial u_{z k}}{\partial z}\right|_{z=h_{k}}=0(k \leq n-1) \\ \left.\sigma_{z k}\right|_{z=h_{k}}=0(k=n)\end{array}\right.$

(2) Friction force and displacement continuity condition at the interface of the $k$ th soil-pile layer :

$R_{k}(z, t)=2 \pi r_{0} \tau_{r z k}\left(r_{0}, z, t\right)$

$u_{z k}\left(r_{0}, z, t\right)=w_{k}(z, t)$

(3) Boundary conditions of the $k$ th pile segment:

(1) At the top of the $k$ th pile segment:

$\frac{\partial w_{k}}{\partial z}-\left.\frac{f_{k}(t)}{E_{p} S}\right|_{z=h_{k}}=0$

where $f_{k}(t)$ is the force that acts on the top of $k$ th pile segment by $k+1$ th pile segment and $f_{n}(t)=f(t)$ at the level of pile head.

(2) At the bottom of the pile:

$\frac{c_{b x k}}{E_{p} S} \frac{\partial w_{k}}{\partial t}+\frac{k_{b x k}}{E_{p} S} w_{k}-\left.\frac{\partial w_{k}}{\partial z}\right|_{z=0}=0$ where $c_{b x 1}=c_{b}, k_{b x 1}=k_{b}$.

(4) Initial conditions of the $k$ th soil-pile layer:

(1) Initial conditions of the $k$ th soil layer:

$\left\{\begin{array}{l}\left.u_{r k}\right|_{(r, z, 0)}=0,\left.\frac{\partial u_{r k}}{\partial t}\right|_{(r, z, 0)}=0 \\ \left.u_{z k}\right|_{(r, z, 0)}=0,\left.\frac{\partial u_{z k}}{\partial t}\right|_{(r, z, 0)}=0\end{array}\right.$

(2) Initial conditions of the $k$ th pile segment:

$\left.w_{k}\right|_{(z, 0)}=0,\left.\frac{\partial w_{k}}{\partial t}\right|_{(z, 0)}=0$

\section{SOLUTION OF THE EQUATIONS}

\subsection{Vibrations of the Soil Layer}

Two potential functions are introduced to decouple the displacements $u_{r k}(r, z, t)$ and $u_{z k}(r, z, t)$ in the Eq. (1) and Eq. (2):

$u_{r k}(r, z, t)=\frac{\partial \phi_{s k}(r, z, t)}{\partial r}+\frac{\partial^{2} \psi_{s k}(r, z, t)}{\partial r \partial z}$

$u_{z k}(r, z, t)=\frac{\partial \phi_{s k}(r, z, t)}{\partial z}-\frac{1}{r} \frac{\partial}{\partial r}\left(r \frac{\partial \psi_{s k}(r, z, t)}{\partial r}\right)$

Let $U_{r k}(r, z, s), U_{z k}(r, z, s), \phi_{s k}(r, z, s)$ and $\psi_{s k}(r, z, s)$ to be the Laplace transform of $u_{r k}(r, z, t), u_{z k}(r, z, t)$, $\phi_{s k}(r, z, t)$ and $\psi_{s k}(r, z, t)$ with respect to $\mathrm{t}$, respectively. Taking the Laplace transform of Eq. (1) and Eq. (2) and combining the initial conditions of the soil layer yield:

Radial direction:

$G_{1 k}^{L}\left(\nabla^{2}+\frac{1}{r^{2}}\right) U_{r k}+2 G_{2 k}^{L} \frac{\partial}{\partial z} \omega_{\theta k}^{L}=\rho_{s k} s^{2} U_{r k}$

Longitudinal direction:

$G_{1 k}^{L} \nabla^{2} U_{z k}-2 G_{2 k}^{L}\left(\frac{\partial}{\partial r}+\frac{1}{r}\right) \omega_{\theta k}^{L}=\rho_{s k} s^{2} U_{z k}$

where $G_{1 k}^{L}=\left(\lambda_{k}+2 \mu_{k}\right)+\left(\lambda_{\kappa}^{\prime}+2 \mu_{\kappa}^{\prime}\right) s$,

$G_{2 k}^{L}=\left(\lambda_{k}+\mu_{k}\right)+\left(\lambda_{k}^{\prime}+\mu_{k}^{\prime}\right) s, \quad \nabla^{2}=\frac{\partial^{2}}{\partial r^{2}}+\frac{1}{r} \frac{\partial}{\partial r}+\frac{\partial^{2}}{\partial z^{2}}$ and

$\omega_{\theta k}^{L}=\frac{1}{2}\left(\frac{\partial U_{z k}}{\partial r}-\frac{\partial U_{r k}}{\partial z}\right)$.

Taking the Laplace transform of Eq. (8) and Eq. (9) yields:

$U_{r k}(r, z, s)=\frac{\partial \phi_{s k}(r, z, s)}{\partial r}+\frac{\partial^{2} \psi_{s k}(r, z, s)}{\partial r \partial z}$

$U_{z k}(r, z, s)=\frac{\partial \phi_{s k}(r, z, s)}{\partial z}-\frac{1}{r} \frac{\partial}{\partial r}\left(r \frac{\partial \psi_{s k}(r, z, s)}{\partial r}\right)$ 
Substituting Eq. (12) and Eq. (13) into Eq. (10) and Eq. (11) yields:

$G_{1 k}^{L} \frac{\partial}{\partial r} \nabla^{2} \phi_{s k}+\left(\mu_{k}+\mu_{k}^{\prime} s\right) \frac{\partial^{2}}{\partial r \partial z} \nabla^{2} \psi_{s k}=\rho_{s k} s^{2}\left(\frac{\partial \phi_{s k}}{\partial r}+\frac{\partial^{2} \psi_{s k}}{\partial r \partial z}\right)$

$G_{1 k}^{L} \frac{\partial}{\partial z} \nabla^{2} \phi_{s k}-\left(\mu_{k}+\mu_{k}^{\prime} s\right)\left(\frac{\partial^{2}}{\partial r^{2}}+\frac{1}{r} \frac{\partial}{\partial r}\right) \nabla^{2} \psi_{s k}$

$=\rho_{s k} s^{2}\left[\frac{\partial \phi_{s k}}{\partial z}-\left(\frac{\partial^{2}}{\partial r^{2}}+\frac{1}{r} \frac{\partial}{\partial r}\right) \psi_{s k}\right]$

And thus Eq. (14) and Eq. (15) can be decoupled as follows:

$\nabla^{2} \phi_{s k}-\frac{s^{2}}{v_{l k}^{2}} \phi_{s k}=0$

$\nabla^{2} \psi_{s k}-\frac{s^{2}}{v_{s k}^{2}} \psi_{s k}=0$

where $v_{l k}=\sqrt{\frac{G_{1 k}^{L}}{\rho_{s k}}}, \quad v_{s k}=\sqrt{\frac{\mu_{k}+\mu_{k}^{\prime} s}{\rho_{s k}}}, \quad \xi_{k}=\sqrt{\frac{2\left(1-v_{k}\right)}{1-2 v_{k}}} ;$

$V L_{k}=\sqrt{\frac{\lambda_{k}+2 \mu_{k}}{\rho_{s k}}}$ and $V S_{k}=\sqrt{\frac{\mu_{k}}{\rho_{s k}}}$ are longitudinal wave and shear wave velocity of the $k$ th soil layer, respectively.

Solving Eq. (16) and Eq. (17) with method of separation of variables first and then combining Eq. (12) and Eq. (13), we can obtain the solutions of $U_{r k}(r, z, s)$ and $U_{z k}(r, z, s)$ as follows:

$$
\begin{aligned}
U_{r k}(r, z, s)= & {\left[A_{1 k} \cos \left(\beta_{k} z\right)+B_{1 k} \sin \left(\beta_{k} z\right)\right] \cdot } \\
& {\left[C_{1 k} I_{1}\left(\eta_{k} r\right)-D_{1 k} K_{1}\left(\eta_{k} r\right)\right] \eta_{k}-} \\
& {\left[A_{2 k} \sin \left(\beta_{k} z\right)-B_{2 k} \cos \left(\beta_{k} z\right)\right] \cdot } \\
& {\left[C_{2 k} I_{1}\left(s_{k} r\right)-D_{2 k} K_{1}\left(s_{k} r\right)\right] \beta_{k} s_{k} } \\
U_{z k}(r, z, s)= & {\left[B_{1 k} \cos \left(\beta_{k} z\right)-A_{1 k} \sin \left(\beta_{k} z\right)\right] \cdot } \\
& {\left[C_{1 k} I_{0}\left(\eta_{k} r\right)+D_{1 k} K_{0}\left(\eta_{k} r\right)\right] \beta_{k} } \\
& -\left[A_{2 k} \cos \left(\beta_{k} z\right)+B_{2 k} \sin \left(\beta_{k} z\right)\right] \cdot \\
& {\left[C_{2 k} I_{0}\left(s_{k} r\right)+D_{2 k} K_{0}\left(s_{k} r\right)\right] s_{k}^{2} }
\end{aligned}
$$

where $I_{0}()$ and $K_{0}()$ are modified Bessel functions of order zero of the first and second kind, respectively; $I_{1}()$ and $K_{1}()$ are modified Bessel functions of order first of the first and second kind, respectively. $A_{1 k}, B_{1 k}, C_{1 k}, D_{1 k}, A_{2 k}, B_{2 k}$, $C_{2 k}, D_{2 k}$ and $\beta_{k}$ are constants to be determined by the boundary conditions. $\eta_{k}$ and $s_{k}$ satisfy the following relationship:

$$
\begin{aligned}
& \beta_{k}^{2}-\eta_{k}{ }^{2}=-\frac{s^{2}}{v_{l k}^{2}} \\
& \beta_{k}^{2}-s_{k}{ }^{2}=-\frac{s^{2}}{v_{s k}^{2}}
\end{aligned}
$$

For convenience, repeat Eq. (4-a) here:

$\left\{\begin{array}{l}\left.u_{r k}(r, z, t)\right|_{r \rightarrow \infty} \rightarrow 0 \\ \left.u_{z k}(r, z, t)\right|_{r \rightarrow \infty} \rightarrow 0\end{array}\right.$

Take the Laplace transform of Eq. (22):

$$
\left\{\begin{array}{l}
\left.U_{r k}(r, z, s)\right|_{r \rightarrow \infty} \rightarrow 0 \\
\left.U_{z k}(r, z, s)\right|_{r \rightarrow \infty} \rightarrow 0
\end{array}\right.
$$

Similarly, taking the Laplace transform of Eq.(4-b) to (4d) as follows:

$$
\begin{aligned}
& U_{r k}\left(r_{0}, z, s\right)=0 \\
& \left(\frac{k_{s x k}}{E_{s k}}+\frac{c_{s x k}}{E_{s k}} s\right) U_{z k}(r, z, s)-\left.\frac{\partial U_{z k}(r, z, s)}{\partial z}\right|_{z=0}=0 \\
& \left\{\begin{array}{c}
\left(\frac{k_{s s k}}{E_{s k}}+\frac{c_{s s k}}{E_{s k}} s\right) U_{z k}(r, z, s)+\left.\frac{\partial U_{z k}(r, z, s)}{\partial z}\right|_{z=h_{k}}=0 \\
{\left[\left(\lambda_{n}+2 \mu_{n}\right)+\left(\lambda_{n}^{\prime}+2 \mu_{n}^{\prime}\right) s\right] \frac{\partial U_{z n}}{\partial z}+\left.\left(\lambda_{n}+\lambda_{n}^{\prime} s\right) \frac{\partial\left(r U_{r n}\right)}{r \partial r}\right|_{z=h_{n}}=0}
\end{array}\right.
\end{aligned}
$$

Substituting Eq. (18) and Eq. (19) into Eq. (4-a') to (4-d') and solving them simultaneously yields $U_{r k}(r, z, s)$ and $U_{z k}(r, z, s):$

$\left\{\begin{array}{l}U_{r k}(r, z, s)=\sum_{m=1}^{\infty} a_{k m} M_{k m} \sin \left(\beta_{k m} z-\theta_{k m}\right) \eta_{k m}\left[K_{1}\left(\eta_{k m} r\right)-\frac{K_{1}\left(\eta_{k m} r_{0 k}\right)}{K_{1}\left(s_{k m} r_{0 k}\right)} K_{1}\left(s_{k m} r\right)\right] \\ U_{z k}(r, z, s)=\sum_{m=1}^{\infty} a_{k m} M_{k m} \cos \left(\beta_{k m} z-\theta_{k m}\right)\left[\frac{\eta_{k m} K_{1}\left(\eta_{k m} r_{0 k}\right)}{\beta_{k m} K_{1}\left(s_{k m} r_{0 k}\right)} s_{k m} K_{0}\left(s_{k m} r\right)-\beta_{k m} K_{0}\left(\eta_{k m} r\right)\right]\end{array}\right.$

where $a_{k m}$ is a constants to be determined. $\beta_{k m}, \eta_{k m}, s_{k m}$, $\theta_{k m}$ and $M_{k m}$ can be obtained from the following equations:

$\tan \left(\beta_{k m} h_{k}\right)=\frac{\left(K X_{k}+K S_{k}\right) \beta_{k m}}{\beta_{k m}^{2}-K X_{k} \times K S_{k}}$

$\beta_{k m}{ }^{2}-\eta_{k m}{ }^{2}=-\frac{s^{2}}{v_{l k}^{2}}$

$\beta_{k m}{ }^{2}-s_{k m}{ }^{2}=-\frac{s^{2}}{V_{s k}^{2}}$

$\tan \left(\theta_{k m}\right)=\frac{K X_{k}}{\beta_{k m}}$

$M_{k m}=\sqrt{1+\left(\frac{\beta_{k m}}{K X_{k}}\right)^{2}}$

where $\left\{\begin{array}{l}K S_{k}=\frac{k_{s s k}+c_{s s k} s}{E_{s k}}(k<n) \\ K S_{k}=0(k=n) \\ K X_{k}=\frac{k_{s x k}+c_{s x k} s}{E_{s k}}\end{array}\right.$ 


\subsection{Solution for Longitudinal Vibrations of the Pile}

Denote $W_{k}(z, s)$ as the Laplace transform with respect to time of $w_{k}(z, t)$ and take the Laplace transform of Eq. (3). After using the initial condition (7-b), we can get:

$\frac{d^{2} W_{k}(z, s)}{d z^{2}}+p^{2} W_{k}(z, s)=-\frac{R_{k}(z, s)}{E_{p} S}$

where $p^{2}=-\frac{s^{2}}{V P^{2}}$.

Rewrite boundary condition (5-a) as :

$$
\begin{aligned}
R_{k}(z, s)= & 2 \pi r_{0}\left(\mu_{k}+\mu_{k}^{\prime} s\right) . \\
& \sum_{m=1}^{\infty} a_{k m} M_{k m} \cos \left(\beta_{k m} z-\theta_{k m}\right)\left(\beta_{k m}^{2}-s_{k m}^{2}\right) \frac{\eta_{k m}}{\beta_{k m}} K_{1}\left(\eta_{k m} r_{0}\right)
\end{aligned}
$$

Then solving Eq. (30) for $W_{k}$ and using boundary condition Eq.(31) gives:

$W_{k}=A \sin (p z)+B \cos (p z)+\sum_{m=1}^{\infty} C_{k m} \cos \left(\beta_{k m} z-\theta_{k m}\right)$

where $\left\{\begin{array}{l}C_{k m}=C_{k m}^{\prime} a_{k m} \\ C_{k m}^{\prime}=\frac{2 \pi r_{0}\left(\mu_{k}+\mu_{k}^{\prime} s\right) M_{k m}\left(s_{k m}^{2}-\beta_{k m}^{2}\right) \eta_{k m} K_{1}\left(\eta_{k m} r_{0}\right)}{E_{p} S\left(p^{2}-\beta_{k m}^{2}\right) \beta_{k m}}\end{array}\right.$

Taking the Laplace transform of Eq. (5-b) gives $U_{z k}\left(r_{0}, z, s\right)=W_{k}(z, s)$, which can be further rewritten as follows:

$\sum_{m=1}^{\infty} a_{k m} D_{k m} \cos \left(\beta_{k m} z-\theta_{k m}\right)$

$=\sum_{m=1}^{\infty} C_{k m} \cos \left(\beta_{k m} z-\theta_{k m}\right)+A \sin (p z)+B \cos (p z)$

where $D_{k m}=-M_{k m}\left[\beta_{k m} K_{0}\left(\eta_{k m} r_{0}\right)-\frac{\eta_{k m} K_{1}\left(\eta_{k m} r_{0}\right)}{\beta_{k m} K_{1}\left(s_{k m} r_{0}\right)} s_{k m} K_{0}\left(s_{k m} r_{0}\right)\right]$

It can be proved that $\cos \left(\beta_{k m} z-\theta_{k m}\right)$ form an orthogonal set over the interval $\left[0, h_{k}\right]$ as follow:

$\left\{\begin{array}{l}\int_{0}^{h_{k}} \cos \left(\beta_{k m} z-\theta_{k m}\right) \cos \left(\beta_{k n} z-\theta_{k n}\right) d z \neq 0(m=n) \\ \int_{0}^{h_{k}} \cos \left(\beta_{k m} z-\theta_{k m}\right) \cos \left(\beta_{k n} z-\theta_{k n}\right) d z=0(m \neq n)\end{array}\right.$

Multiplying both sides of Eq. (34) by $\frac{2}{h_{k}} \cos \left(\beta_{k m} z-\theta_{k m}\right)$ and then integrating over the interval $\left[0, h_{k}\right]$, we can get $W_{k}$ :

$$
\begin{aligned}
W_{k} & =\left[\sum_{m=1}^{\infty} \frac{F_{k 1 m} C_{k m}^{\prime} \cos \left(\beta_{k m} z-\theta_{k m}\right)}{\left(D_{k m}-C_{k m}^{\prime}\right) F_{k 3 m}}+\sin (p z)\right] A \\
& +\left[\sum_{m=1}^{\infty} \frac{F_{k 2 m} C_{k m}^{\prime} \cos \left(\beta_{k m} z-\theta_{k m}\right)}{\left(D_{k m}-C_{k m}^{\prime}\right) F_{k 3 m}}+\cos (p z)\right] B
\end{aligned}
$$

$$
\text { where }\left\{\begin{array}{l}
F_{k 1 m}=\frac{2}{h_{k}} \int_{0}^{h_{k}} \sin (p z) \cos \left(\beta_{k m} z-\theta_{k m}\right) d z \\
F_{k 2 m}=\frac{2}{h_{k}} \int_{0}^{h_{k}} \cos (p z) \cos \left(\beta_{k m} z-\theta_{k m}\right) d z \\
F_{k 3 m}=\frac{2}{h_{k}} \int_{0}^{h_{k}} \cos \left(\beta_{k m} z-\theta_{k m}\right) \cos \left(\beta_{k m} z-\theta_{k m}\right) d z
\end{array}\right.
$$

Denoting $F_{k}(s)$ as the Laplace transform of $f_{k}(t)$ with respect to $\mathrm{t}$ and taking the Laplace transform of the boundary conditions Eq. (6-a) and Eq. (6-b) yields:

$$
\begin{aligned}
& \frac{d W_{k}}{d z}-\left.\frac{F_{k}(s)}{E_{p} S}\right|_{z=h_{k}}=0 \\
& E_{p} S \frac{d W_{k}}{d z}=\left.\left(k_{b x k}+c_{b x k} s\right) W_{k}\right|_{z=0}
\end{aligned}
$$

Substituting Eq. (37) into Eq. (6-a') to (6-b') and solving for $A$ and $B$, we can obtain the impedance function at the $k$ th pile segment head:

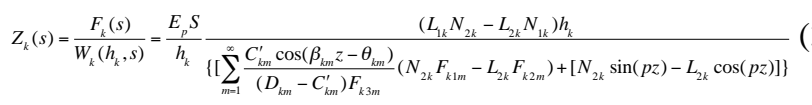

where $L_{1 k}=-\sum_{m=1}^{\infty} \frac{F_{k 1 m} C_{k m}^{\prime} \beta_{k m}}{\left(D_{k m}-C_{k m}^{\prime}\right) F_{k 3 m}} \sin \left(\beta_{k m} h_{k}-\theta_{k m}\right)+p \cos \left(p h_{k}\right)$,

$$
N_{1 k}=-\sum_{m=1}^{\infty} \frac{F_{k 2 m} C_{k m}^{\prime} \beta_{k m}}{\left(D_{k m}-C_{k m}^{\prime}\right) F_{k 3 m}} \sin \left(\beta_{k m} h_{k}-\theta_{k m}\right)-p \sin \left(p h_{k}\right),
$$

$$
L_{2 k}=\sum_{m=1}^{\infty} \frac{C_{k m}^{\prime} F_{k 1 m}\left[\frac{Z_{k-1}}{E_{p} S} \cos \left(\theta_{k m}\right)-\beta_{k m} \sin \left(\theta_{k m}\right)\right]}{\left(D_{k m}-C_{k m}^{\prime}\right) F_{k 3 m}}-p,
$$

$N_{2 k}=\sum_{m=1}^{\infty} \frac{C_{k m}^{\prime} F_{k 2 m}\left[\frac{Z_{k-1}}{E_{p} S} \cos \left(\theta_{k m}\right)-\beta_{k m} \sin \left(\theta_{k m}\right)\right]}{\left(D_{k m}-C_{k m}^{\prime}\right) F_{k 3 m}}+\frac{Z_{k-1}}{E_{p} S}$.

And then the velocity admittance function at the $k$ th pile segment can be obtained as follows:

$G_{v k}(s)=\frac{s}{Z_{k}(s)}$

Set $i=\sqrt{-1}, \omega$ and $T_{k}=\frac{h_{k}}{V P}$ to be the imaginary unit, the circular frequency and the propagating time in the $k$ th pile segment, respectively. For convenience, some dimensionless parameters are introduced as follows:

$$
\begin{gathered}
\overline{r_{0 k}}=\frac{r_{0}}{h_{k}} ; \quad \overline{\rho_{k}}=\frac{\rho_{s k}}{\rho_{p}} ; \quad v_{s p k}=\frac{V S_{k}}{V P} ; \quad \gamma_{k}=\frac{2 v_{s p k}^{2} \overline{\rho_{k}}}{\overline{r_{0 k}}} ; \quad \overline{p_{k}}=\omega T_{k} ; \\
D_{\mu}=\frac{\mu_{k}^{\prime}}{\mu_{k} T_{k}} ; \quad D_{\lambda}=\frac{\lambda_{k}^{\prime}}{\lambda_{k} T_{k}} ; \quad R_{x k}=\frac{k_{s x k} h_{k}}{E_{s k}} ; \quad I_{x k}=\frac{c_{s x k} h_{k}}{E_{s k} T_{k}} ;
\end{gathered}
$$




$$
\begin{array}{ll}
\overline{K X_{k}}=\left(R_{x k}+i I_{x k} \overline{p_{k}}\right) ; R_{s k}=\frac{k_{s s k} h_{k}}{E_{s k}} ; I_{s k}=\frac{c_{s s k} h_{k}}{E_{s k} T_{k}} ; \overline{\beta_{k}}=\beta_{k} h_{k} ; & \tan \left(\overline{\beta_{k}}\right)=\frac{\left[\overline{K X_{k}}+\overline{K S_{k}}\right] \overline{\beta_{k}}}{\left[\overline{\beta_{k}^{2}}-\overline{K X_{k}} \overline{K S_{k}}\right]} \\
\overline{K S_{k}}=\left(R_{s k}+i I_{s k} \overline{p_{k}}\right) ; & a_{s k}=\frac{\omega h_{k}}{V S_{k}} ; M_{k m}=\sqrt{1+\left(\overline{\overline{\beta_{k m}}}\right)^{2}} ; \quad \tan \left(\theta_{k m}\right)=\frac{\overline{K X_{k}}}{\overline{\beta_{k m}}}
\end{array}
$$

where equation (41) is a complex transcendental equation and can be solved by numerical method.

Substituting $s=i \omega$ into Eq. (39) to (40), the displacement impedance function and velocity admittance at the head of $k$ th pile segment can be expressed as follows:

$$
\begin{aligned}
& K_{d k}=\frac{E_{p} S}{h_{k}} K_{d k}^{\prime} \\
& H_{v k}(\omega)=\frac{1}{\rho_{p} S \cdot V P} H_{v l k}
\end{aligned}
$$

where $K_{d k}^{\prime}$ and $H_{v l k}$ are dimensionless displacement impedance function and velocity admittance function, respectively:

$$
\begin{aligned}
& K_{d k}^{\prime}=\overline{Z_{k}}=\frac{\left(\overline{L_{1 k}} \overline{N_{2 k}}-\overline{L_{2 k} N_{1 k}}\right)}{\overline{D N_{k}}} \\
& H_{v l k}=\frac{1}{\overline{Z_{k}}} i \overline{p_{k}}
\end{aligned}
$$

Set $k=n$ in Eq. (41) and Eq. (42) and then the displacement impedance function and velocity admittance at the pile head can be obtained.

When the excitation acting on the pile head is a half-sine pulse such as $f(t)=Q_{\max } \sin \left(\frac{\pi}{T} t\right), \quad t \in(0, T)$, where $T$ denotes the impulse width, then the semi-analytical velocity response of the pile head can be expressed as:

$$
\begin{aligned}
& g_{n}(t)=Q_{\max } I F T\left(H_{v n} \frac{\pi T}{\pi^{2}-T^{2} \omega^{2}}\left(1+e^{-i \omega T}\right)\right)=\frac{Q_{\max }}{\rho_{p} S \cdot V P} g_{n}^{\prime}(t) \\
& g_{n}^{\prime}(t)=\frac{1}{2 \pi} \int_{-\infty}^{\infty} H_{v \ln } \frac{\pi T^{\prime}}{\pi^{2}-T^{\prime 2} \bar{\omega}^{2}}\left(1+e^{-i \bar{\omega} T^{\prime}}\right) e^{i \bar{\omega} \omega^{\prime}} d \bar{\omega}
\end{aligned}
$$

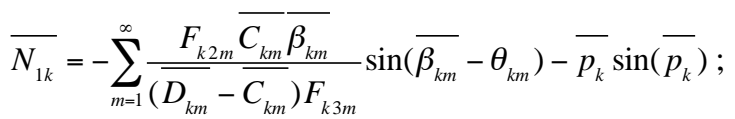

$\overline{N_{2 k}}=\sum_{m=1}^{\infty} \frac{\overline{C_{k m}} F_{k 2 m}\left[\overline{Z_{k-1}} \cos \left(\theta_{k m}\right)-\overline{\beta_{k m}} \sin \left(\theta_{k m}\right)\right]}{\left(\overline{D_{k m}}-\overline{C_{k m}}\right) F_{k 3 m}}+\overline{Z_{k-1}} ;$

$\overline{D N_{k}}=\overline{N_{2 k}}\left[\sum_{m=1}^{\infty} \frac{F_{k 1 m} \overline{C_{k m}} \cos \left(\overline{\beta_{k m}}-\theta_{k m}\right)}{\left(\overline{D_{k m}}-\overline{C_{k m}}\right) F_{k 3 m}}+\sin \left(\overline{p_{k}}\right)\right]$

$-\overline{L_{2 k}}\left[\sum_{m=1}^{\infty} \frac{F_{k 2 m} \overline{C_{k m}} \cos \left(\overline{\beta_{k m}}-\theta_{k m}\right)}{\left(\overline{D_{k m}}-\overline{C_{k m}}\right) F_{k 3 m}}+\cos \left(\overline{p_{k}}\right)\right]$

where $\overline{\beta_{k}}$ and $\theta_{k m}$ can be determined utilizing the following equations:

longitudinal wave propagating from the pile head to pile tip. $t^{\prime}=\frac{t}{T_{c}}, \quad T^{\prime}=\frac{T}{T_{c}}$ and $\bar{\omega}=\omega T_{c}$ denote the dimensionless time, dimensionless impulse width and dimensionless circular frequency, respectively.

\section{PARAMETRIC STUDY AND DISCUSSION}

In the following, the effects of variety of soil modulus on the velocity admittance curves and reflection wave curves 
are discussed, which are the theoretical basis of mechanical impedance analysis method and reflection wave method. For convenience, the subscripts marking the soil layer are eliminated if the parameter values are same in each layer in the following figures. Number sequence of the soil layer is shown in Fig. (1). Parameters using in the analysis are shown as follows:

$V_{i j}=V S_{i} / V S_{j} 、 V P=3500 、 r_{0}=0.5 、 \rho_{p}=2500$ 、

$c_{b}=5 \times 10^{5} 、 k_{b}=10^{9} 、 v=0.4 、 v^{\prime}=0.4 、 \mu^{\prime}=5 \times 10^{3}$ 、

$\bar{\rho}=0.7 、 \frac{k_{s x k}}{E_{s k}}=1 、 c_{s x k}=10^{4} 、 \frac{k_{s s k}}{E_{s k}}(k<n)=1$ 、

$c_{s x k}(k<n)=10^{4} 、 T=1.5 \mathrm{~ms}$.

The case of two soil layers: Influence of modulus of upper soil layer on the velocity admittance curve and reflection wave curve at the pile top

Fig. (3.a) shows that the variation of modulus of the upper soil layer has significant effect on the velocity admittance curve. Compared with the case that the soil is homogeneous, the velocity admittance curve of the pile top has a phenomenon that there is a smaller peak between every two adjacent larger peaks. When the upper soil layer is stiffer than the lower soil layer, it can be seen that the amplitude of the velocity admittance increase at first as the frequency increases, but then decrease as the frequency further increases after the amplitude is beyond the maximum. When the upper soil layer is softer than the lower soil layer, it can be seen that the amplitude of the velocity admittance decrease as the frequency increases and the amplitude increase as the frequency further increases after the amplitude is beyond the minimum. For two cases, the length between every two adjacent larger peaks (large peak cycle) is almost equal, which reflects the location where the soil impedance varies abruptly with the relationship $h_{2} \approx V P \cdot \pi / d \omega_{\max }=10.18 \mathrm{~m}$. The length between one larger peak and its corresponding adjacent smaller peak (smaller peak cycle) reflects the pile length with the relationship $H \approx V P \cdot \pi / d \omega=19.64 m$. Fig. (3.b) shows that wave curve at the division surface concaves and is out of phase with the input pulse when the upper soil layer is stiffer than the lower soil layer. The wave curve at the division surface convexes and is in phase with the input pulse when the upper soil layer is softer than the lower soil layer.

Fig. (4.a) and Fig. (4.b) show that the amplitude of the velocity admittance decreases as the modulus of the upper soil layer increases and oscillation is weak. The dynamic stiffness at the low frequency range increases and the amplitude of the input impulse and the reflection amplitude at the pile tip decreases. The amplitude of the reflected wave amplitude at the division surface increases. It is due to more energy dissipation in the shallow layer as the modulus of the upper soil layer increases.

The case of three soil layers: Influence of modulus of surrounding soil on the velocity admittance curve and reflection wave curve at the pile top $\mathbf{a}$

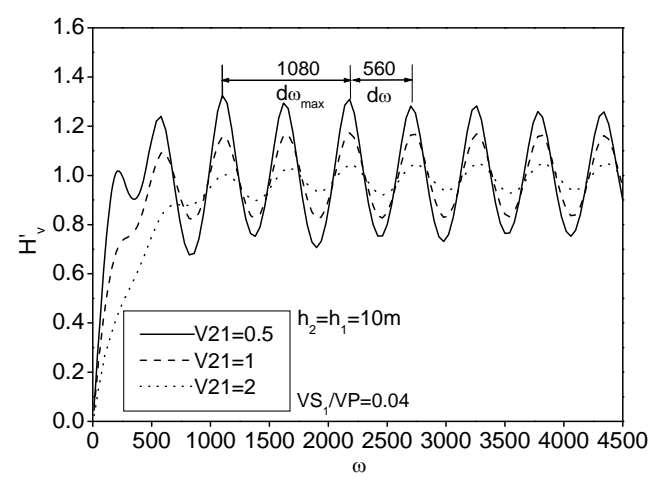

b

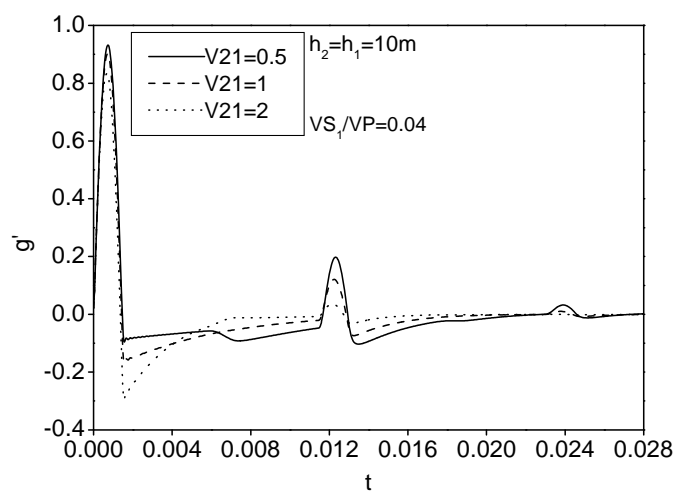

Fig. (3). Influence of Variations of modulus of upper soil layer on velocity admittance curve and reflection wave curve.

a

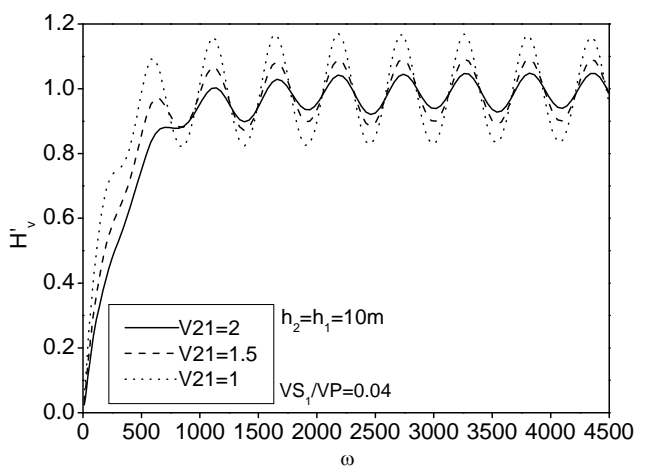

b

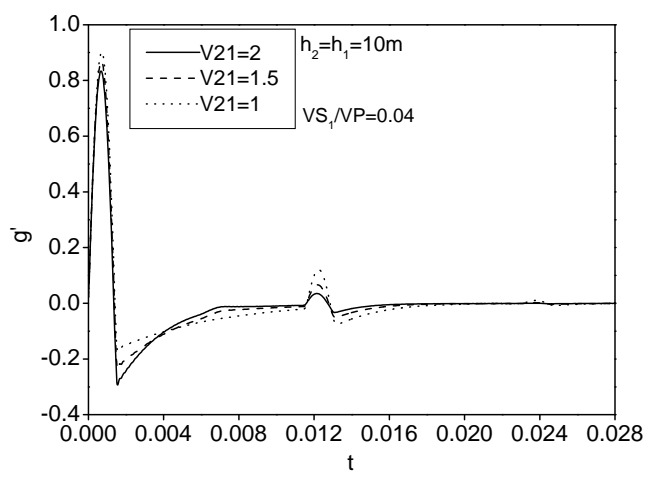

Fig. (4). Influence of varying degree of modulus of upper soil layer on velocity admittance curve and reflection wave curve. 
Fig. (5.a) and Fig. (5.b) show that the velocity admittance curve is similar to the case of two soil layers when there is stiffer or softer interlayer in the surrounding soil. Large peak cycle reflects the location of the interlayer with the relationship $h_{3} \approx V P \cdot \pi / d \omega_{\max }=6.5 \mathrm{~m}$. At the first peak, the amplitude of the case with stiffer interlayer is larger than that of the case with homogeneous soil and the amplitude of the case with softer interlayer is smaller than that of the case with homogeneous soil. Consequently, the properties of the upper soil layer have significant effect on the amplitude of the first peak. The wave curve at the division surface is out of phase with the input pulse for stiffer interlayer case and in phase with the input pulse for softer interlayer case. The amplitude of the reflection wave at the pile tip decrease as the modulus of the stiffer or softer interlayer increases.

a

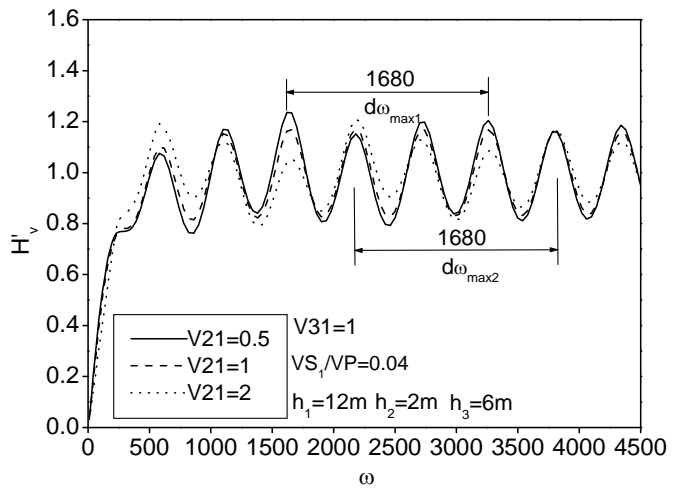

b

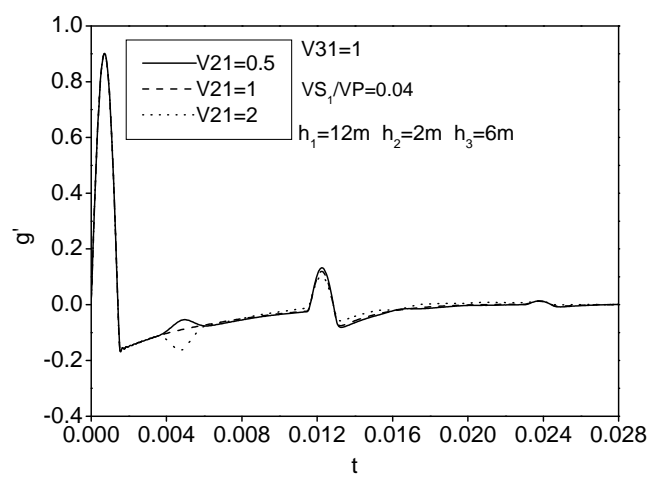

Fig. (5). Influence of hard or soft interlayer on velocity admittance curve and reflection wave curve.

Fig. (6.a) and Fig. (6.b) show that the occurring time of the reflected waves of the interlayer is retarded as the buried depth of the softer layer becomes deeper. The large peak cycle of the velocity admittance curve reflects the buried depth of the interlayer with the relationship $h_{3} \approx V P \cdot \pi / d \omega_{\max 1} / d \omega_{\max 2} / d \omega_{\max 3}=6.39 \mathrm{~m} / 8.46 \mathrm{~m} / 10.18 \mathrm{~m}$.

Fig. (7.a) and Fig. (7.b) show that the amplitude of the velocity admittance increases but the reflection amplitude of the interlayer tends to be weaker as the thickness of the softer interlayer increases. The reflection amplitude at the pile tip increase. It is due to less energy dissipation in the interlayer as its thickness increases. $\mathbf{a}$

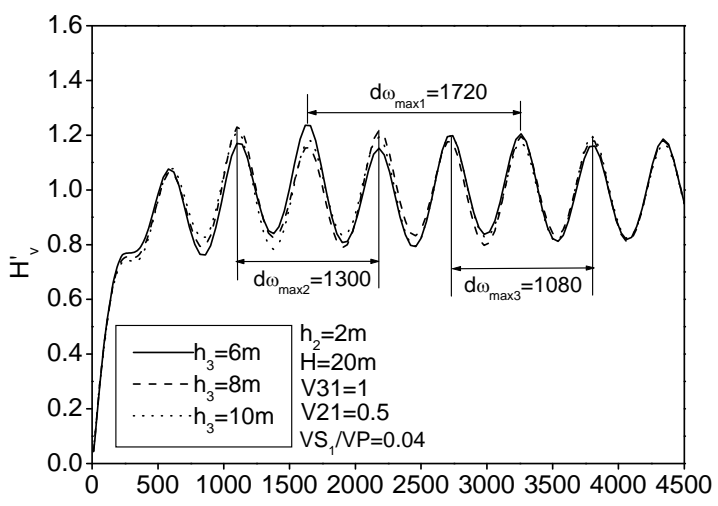

$\omega$

b

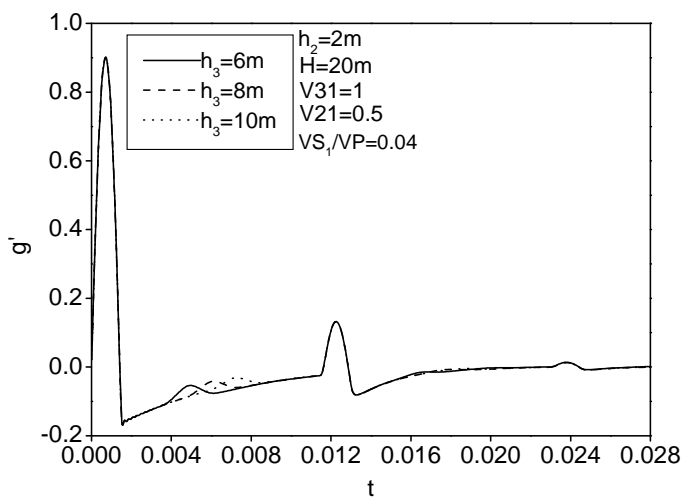

Fig. (6). Influence of location of soft interlayer on velocity admittance curve and reflection wave curve.

a

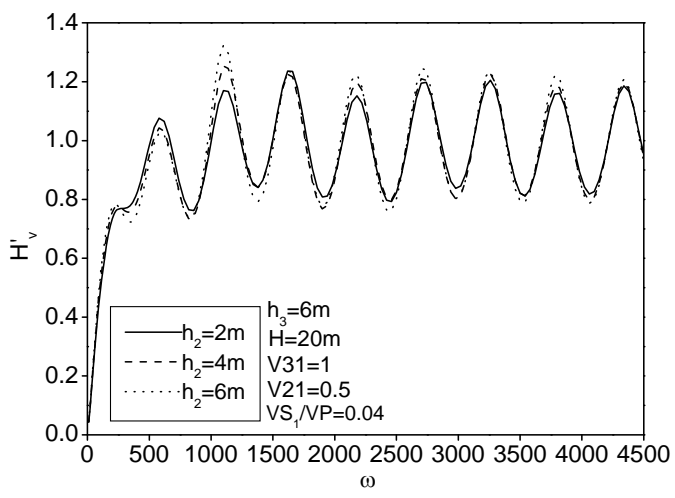

b

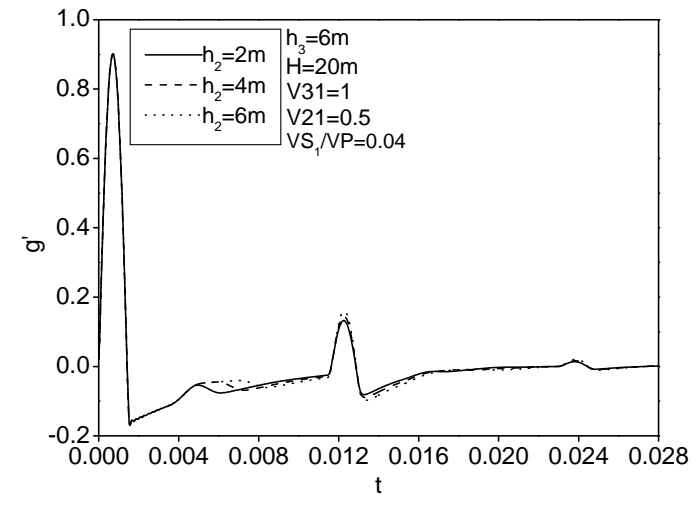

Fig. (7). Influence of length of soft interlayer on velocity admittance curve and reflection wave curve. 


\section{CONCLUSIONS}

(1) Modeling surrounding soil as a three-dimensional axisymmetric continuum and considering its wave effect, soil-pile dynamic longitudinal interaction in viscous layered soils is studied. The analytical solution of mobility in the frequency domain and semi-analytical solution of velocity response in the time domain undergoing a half-cycle sine pulse force have been derived. Based on the solutions herein, the effect of stiffer or softer interlayer on the mobility curves and reflection wave curves of integrated pile is studied

(2) Compared with the case of homogeneous soil, there is a smaller peak between every two adjacent larger peaks on the mobility curve in layered soil. The difference between two adjacent larger peaks can be used to locate where abrupt variety of soil modulus happens. At the division surface, the reflection wave curve is out of phase with the input pulse if soil modulus increases and in phase with the input pulse if soil modulus decreases.

\section{REFERENCES}

[1] K. H. Van, P. Middendorp, and B. P Van, "An analysis of dissipative wave propagation in a pile", Intl seminar on the application of Stress-Wave Theory onpiles/Stockholm, 1980.

[2] D. W. Chang and S. H. Yeh, "Time-domain wave equation analyses of single piles utilizing tansformed radiation damping", Soils and Foundations, JGS, vol. 39, no.2, pp. 31- 44, 1999.

[3] W. Teng, W. Kuihua, and X. Kanghe, "Study on vibration properties of piles in layered soils", China Civil Engineering Journal, vol. 35, no. 1, pp. 83-87, 2001. (in Chinese).
[4] L. Dongjia, "Dynamic axial response of multi-defective piles in nonhomogeneous soil", Chinese Journal of Geotechnical Engineering, vol. 22, no. 4, pp. 391-395, 2002. (in Chinese).

[5] W. Kuihua, "Vibration of inhomogeneous viscous-elastic pile embedded in layered soils with general Voigt model", Journal of Zhejiang University (Engineering Science), vol. 36, no. 5, pp. 565571, 595, 2002. (in Chinese).

[6] W. Kuihua and Y. Hongwei, "Vibration of inhomogeneous pile embedded in layered soils with general Voigt model", Acta Mechanica Solida Sinica, vol. 24, no. 3, pp. 293-303, 2003. (in Chinese).

[7] F. Shijin, C. Yunmin, and L. Mingzhen, "Analysis and application in engineering on vertical vibration of viscoelasticity piles in layered soil", China Journal of Highway and Transport, vol. 17, no. 2, pp. 59-63, 2004. (in Chinese).

[8] T. Nogami and M. Novak, "Soil-pile interaction in vertical vibration", Earthquake Engineering and Structural Dynamics, vol. 4, pp. 277-293, 1976.

[9] M. Novak and F. Aboul-Ella, "Impedance functions of piles in layered media", Journal of the Engineering Mechanics Division, $A S C E$, vol. 104, pp. 643-661, 1978.

[10] M. Novak, T. Nogami, and F. Aboul-Ella, "Dynamic soil reactions for plane strain case", Journal of the Engineering Mechanics Division, ASCE, vol.104 (EM4), pp. 953-959, 1978.

[11] G. Militano and R. K. N. D. Rajapakse, "Dynamic response of a pile in a multi-layered soil to transient torsional and axial loading", Geotechnique, vol. 49, no. 1, pp. 91-109, 1999.

[12] H. Changbin, W. Kuihua, and X. Kanghe, "Time domain analysis of vertical dynamic response of a pile considering the effect of pile-soil interaction", Chinese Journal of Computational Mechanics, vol. 21, no. 4, pp. 392-399, 2004. (in Chinese).

[13] H. Changbin, W. Kuihua, and X. Kanghe, "Time Domain Axial Response of Dynamically Loaded Pile in Viscous Damping Soil Layer", Journal of Vibration Engineering, vol. 17, no.1, pp. 72-77, 2004. (in Chinese).

(C) Wang et al.; Licensee Bentham Open.

This is an open access article licensed under the terms of the Creative Commons Attribution Non-Commercial License (http://creativecommons.org/licenses/_by-nc/3.0/) which permits unrestricted, non-commercial use, distribution and reproduction in any medium, provided the work is properly cited. 